\title{
New-Onset Refractory Status Epilepticus with Underlying Autoimmune Etiology: a Case Report
}

\author{
Lucille Brunker $^{1}$ - Priscilla Hirst ${ }^{2}$ (D) Joseph J. Schlesinger ${ }^{3}$
}

Accepted: 7 November 2019 / Published online: 28 November 2019

(C) Springer Nature Switzerland AG 2019

\begin{abstract}
Management of new-onset refractory status epilepticus and the approach to burst suppression variable is often challenging. We present the unusual case of a previously healthy 18-year-old male with new-onset status epilepticus admitted to the neurologic intensive care unit for 70 days. Despite treatment with multiple anti-epileptic drugs in addition to IV anesthetics, burst suppression was initially unsustainable and the patient remained in super-refractory status epilepticus. Extensive evaluation revealed an underlying autoimmune-mediated etiology with positivity for glutamic acid decarboxylase- 65 antibody. Clinical response with a goal of 1-2 bursts per screen on EEG monitor was eventually achieved after a course of rituximab and plasma exchange therapy as well as a 7-day barbiturate coma with a regimen of clobazam, lacosamide, Keppra, and oxcarbazepine followed by a slow taper of phenobarbital and the addition of fosphenytoin. Remarkably, the patient was subsequently discharged to a rehabilitation facility with complete neurologic recovery. We discuss treatment strategies for new-onset refractory status epilepticus and highlight the role of rapid initiation of burst suppression with high-dose IV anesthetics to ensure neuroprotection while the underlying etiology is addressed with immune-modulating therapy.
\end{abstract}

Keywords Barbiturate coma $\cdot$ Burst suppression $\cdot$ GAD65 $\cdot$ Ketamine $\cdot$ Refractory status epilepticus

\section{Introduction}

The management of first-episode unprovoked seizures involves an individualized approach that weighs the risk of seizure recurrence against the adverse effects of anti-epileptic drugs (AEDs). While sharing a common property of suppressing seizures, AEDs can be classified into different pharmacological subtypes based on the mechanism of action, tendency for drug-drug interactions, and toxicity profiles. Major mechanisms of action include alteration of sodium currents, modulation of GABAergic tone, antagonism of the NMDA

This article is part of the Topical Collection on Medicine

Priscilla Hirst

Priscilla.hirst@mail.mcgill.ca

1 Department of Anesthesiology, Vanderbilt University Medical Center, Nashville, TN, USA

2 Department of Medicine, Montefiore Health System, New Rochelle, United States

3 Department of Anesthesiology, Department of Hearing and Speech Sciences, Department of Biomedical Engineering, Vanderbilt University Medical Center, Nashville, TN, USA glutamate receptor, alteration of calcium currents, and the binding of synaptic vesicle proteins.

Refractory status epilepticus (RSE) is defined as persistent seizures despite administration of an initial benzodiazepine and a nonbenzodiazepine antiseizure drug [1]. Super refractory status epilepticus (SRSE) is defined as status epilepticus that continues or recurs after the onset of anesthetic therapy and that recurs on the reduction of anesthesia [2]. Although the optimal treatment of RSE is unclear in the literature, primary therapies include midazolam, propofol, and phenobarbital. Patients in this setting should be intubated and monitored on continuous electroencephalogram (EEG) with the goal of burst suppression. Of note, prolonged use of a propofol infusion is associated with propofol infusion syndrome (PRIS), which involves rhabdomyolysis, severe metabolic acidosis, as well as cardiac and renal failure, and green urine [3]. In addition, hypotension is common with many patients requiring vasopressor support in this setting [4].

Ketamine is an N-methyl-D-aspartase (NMDA) antagonist that can also be used in the treatment of RSE. Although evidence for its use is limited, a meta-analysis suggests that ketamine contributed to seizure control in RSE for approximately $57 \%$ of adult patients with median loading doses in the range of $1-2 \mathrm{mg} / \mathrm{kg}$, with a continuous infusion ranging 
anywhere from 1 to $10 \mathrm{mg} / \mathrm{kg} / \mathrm{h}$, based on the cited reports [5]. Glutamate antagonists have been shown to be particularly effective in the later phases of SE when GABA agonists have lost some effectiveness and glutamatergic activity may underlie ongoing seizure activity $[6,7]$.

\section{Case description}

A previously healthy 18 -year-old male was admitted to the hospital with new-onset seizures following episodes of staring, drooling, and unresponsiveness in addition to flu-like symptoms and fatigue on the day prior to admission. Possible exposure history was notable for a recent cruise to the Caribbean. En route to the hospital, the patient suffered a generalized tonic-clonic seizure witnessed by EMT.

On initial clinical exam, the patient appeared somnolent although neurologic exam (cranial nerves, motor strength, sensory) was without deficits. Two-hour EEG monitoring revealed continuous $1.5-2-\mathrm{Hz}$ right frontal lateralized periodic discharges. Initial imaging with $\mathrm{CT}$ and MRI was unremarkable.

Inpatient treatment with an AED regimen of levetiracetam and valproic acid was initiated. Given the concern for meningoencephalitis from an infectious etiology, the patient was treated with vancomycin, ceftriaxone, and acyclovir. Over the next $24 \mathrm{~h}$, continuous EEG monitoring was notable for subclinical seizures and the patient was started on oxcarbazepine, lacosamide, and topiramate with as needed lorazepam. Based on the results of lumbar puncture and cerebral spinal fluid analysis, acyclovir and antibiotics were discontinued.

On hospital day 3, the patient was found to be in status epilepticus (SE) on EEG and unresponsive but without convulsions on exam. He was emergently transferred to the neurologic intensive care unit (NICU) and intubated for airway protection. Continuous EEG monitoring displayed SE despite broad AED therapies (levetiracetam, lacosamide, topiramate, oxcarbazepine). For neurologic protection, the patient was initiated on burst suppression therapy, with a target of 1-2 epileptic bursts per screen on EEG monitor. A midazolam infusion was initiated and up-titrated to $60 \mathrm{mg} \mathrm{h}^{-1}$ but failed to achieve burst suppression, and the patient continued to have generalized epileptic discharges on EEG. The patient was subsequently changed to a propofol infusion up to $150 \mathrm{mcg} \mathrm{kg}$ $\min ^{-1}$ with intermittent boluses to maintain burst suppression. Despite the addition of phenobarbital, epileptic discharge activity remained evident on EEG, and events of facial twitching and arm convulsions were observed on exam. Burst suppression was eventually achieved with the addition of a pentobarbital infusion. Given the severity of ongoing epileptic activity, broad-spectrum antibiotic coverage was resumed with the addition of doxycycline to cover tick-borne illness.
Despite this regimen, the patient continued to have epileptic discharges evident on EEG but was not convulsive on exam. A ketamine infusion was subsequently added. Empiric treatment with intravenous immunoglobulin (IVIg) therapy and methylprednisolone for autoimmune and paraneoplastic causes were initiated. In the following days, the patient continued to experience breakthrough seizures evident on EEG and on exam with facial twitching and rhythmic arm movement when pentobarbital was decreased, consistent with SRSE. These ongoing EEG findings with evident seizures when anesthesia was decreased led to further modification of the AED regimen (Fig. 1, timeline of agents used) including the addition of clobazam. Of note, the patient developed diabetes insipidus secondary to the pentobarbital infusion and a vasopressin infusion was administered.

On hospital day 12, sufficient burst suppression was achieved with a regimen of pentobarbital, ketamine, clobazam, lacosamide, levetiracetam, oxcarbazepine, and valproic acid. When the pentobarbital infusion was decreased, EEG continued to be consistent with SE. For this reason, the phenobarbital infusion was continued in order to maintain burst suppression. At this point, the patient underwent a tracheostomy procedure due to prolonged mechanical ventilation requirements. A ketogenic diet was initiated and continued for the duration of hospitalization [8].

In the following days, the patient remained in SRSE with epileptic activity on EEG and with facial twitching on exam. Felbamate was added to the AED regimen of levetiracetam, lacosamide, oxcarbazepine, and clobazam. Plasma exchange (PLEX) therapy was initiated along with hypothermia protocol $[9,10]$. On day four of five of PLEX therapy, hospital day 19, laboratory results from CSF paraneoplastic and autoimmune evaluation ELISA returned positive for anti-glutamic acid decarboxylase-65 (GAD65) antibody $>1: 4800$ as represented in Table 1. After completion of the 5-day course of PLEX therapy, rituximab was added and dosed every 2 weeks, in addition to the existing AED regimen of oxcarbazepine, clobazam, levetiracetam, and topiramate. At this point, the patient continued to have clinical seizures with bilateral epileptic discharge on EEG. A pentobarbital 7-day coma for burst suppression was then initiated.

The patient was loaded with fosphenytoin on hospital day 27. Following loading and maintenance doses, blood serum levels of therapies were verified and phenytoin levels found to be subtherapeutic at $6.7 \mathrm{mcg} \mathrm{mL}^{-1}$ (goal of $10-20 \mathrm{mcg} \mathrm{mL}^{-1}$ ). Phenobarbital levels were found to be supratherapeutic at 76.2 mcg mL ${ }^{-1}$ ( goal of $10-40 \mathrm{mcg} \mathrm{mL}^{-1}$ ). Appropriate medication alterations were made to bring both drugs to appropriate therapeutic levels (phenobarbital at $35.5 \mathrm{mcg} \mathrm{mL}^{-1}$ and phenytoin at $15.9 \mathrm{mcg} \mathrm{mL}^{-1}$ ). While these agents were being titrated, perampanel was initiated on hospital day 35 . On hospital day 38 , when burst suppression was lifted, clinical 
Significant Events Timeline

- $\mathrm{HD}^{*} 0$ : Admitted with seizure, treated with levetiracetam and valproic acid. Initially treated for infectious etiology with vancomycin, ceftriaxone, and acyclovir

- HD1: EEG showed subclinical seizures, oxcarbazepine, lacosamide, and topiramate added to AED** regimen

- HD3: Status epilepticus, transferred to Neurologic Intensive Care Unit, intubated. Initiated midazolam infusion, with addition of propofol infusion with goal of burst suppression.

- HD4: Added phenobarbital

- HD5: Changed infusions to pentobarbital and ketamine to attempt burst suppression

- HD10: Empiric treatment methylprednisolone and IVIG

- HD12: Burst suppression achieved with AED regimen pentobarbital, ketamine, clobazam, lacosamide, leviteracetam, oxcarbazepine, valproic acid

- HD15: PLEX therapy started for 5 day course

- HD19: CSF paraneoplastic and autoimmune evaluation positive for anti-GAD65

- HD20: Rituximab started once every 2 weeks in addition to AED regimen oxcarbazepine, clobazam, levetiracetam, topiramate

- HD21: Continued pentobarbitaol for 7 day burst suppresion

- HD27: Addition fosphenytoin to AED regimen

- HD35: Addition parempanel to AED regimen

- HD38: Burst suppression lifted, clinical seizures resolved and EEG with focal ictal discharge

- HD44: Focal ictal discharge on EEG resolved

- HD70: Patient discharged to inpatient rehabilitation with final AED regimen

- Phenytoin $200 \mathrm{mg}$ by mouth twice daily

- Phenobarbital $30 \mathrm{mg}$ by mouth four times daily

- Parempanel $12 \mathrm{mg}$ by mouth daily

- Topiramate $200 \mathrm{mg}$ by mouth twice daily

"HD: hospital day

"AED: Anti epileptic drug

Fig. 1 Drug timeline. Given the acute changes and multiple therapies constantly being titrated during a prolonged hospital course, the significant events and therapies are summarized

seizures had resolved. EEG monitoring was continued and, by hospital day 44, focal ictal discharges had ceased.

Throughout the remainder of the admission, the patient was successfully weaned off of the ventilator, was able to tolerate eating meals by mouth, and worked with occupational and physical therapy to return to his baseline functional status. $\mathrm{He}$ was transitioned from fosphenytoin to phenytoin extended release $200 \mathrm{mg}$ by mouth twice daily, continued on phenobarbital $30 \mathrm{mg}$ by mouth four times daily, perampanel $12 \mathrm{mg}$ by mouth each evening, and topiramate $200 \mathrm{mg}$ by mouth twice daily, and ultimately discharged on this AED regimen. The patient was discharged to inpatient rehabilitation to continue recovering from prolonged hospitalization prior to returning home.

\section{Discussion}

Here, we present a case of new-onset SRSE (NORSE) with underlying autoimmune etiology in a previously healthy young male who ultimately made complete neurological recovery after extensive treatment and NICU admission. The clinical outcome raises discussion as NORSE is typically considered a life-threatening condition with mortality up to $40 \%$ [11]. Although general expert consensus in the literature recommends approaching NORSE with pharmacologic-induced coma and continuous infusion of IV anesthetic agents to suppress brain activity and preserve normal brain physiology [2], a precise roadmap to clinical neurological recovery is not described, pointing to educational value in individually described cases. The unusual outcome demonstrated here raises a discussion of optimal approach and treatment as applied to a case of NORSE where complete neurological recovery was ultimately achieved.

In this particular case of NORSE, the underlying etiology was obscure despite an exhaustive workup and was ultimately discovered to be an autoimmune-mediated epilepsy with antiGAD65 antibody positivity. The patient's CSF was tested for a panel of other antibodies against surface antigens relevant to autoimmune etiology, all of which were found to be negative with the exception of GAD65. While positivity of antiGAD65 antibodies should not in itself be considered a cause of autoimmune encephalitis, the other clinical and laboratory findings in the case were also consistent with autoimmune etiology. These findings included a negative infectious workup, a negative paraneoplastic workup, the viral-type symptom prodrome at presentation, and the intractable resistance to AED therapy. On review of the literature, management of SE in setting of known anti-GAD65 antibody positivity is often difficult and, in some cases, irreversible [12, 13]. Case reports described by Mäkelä and colleagues (2018) have shown moderate responses to steroids, IVIg, and PLEX, but immunosuppression with better CNS penetration has been 
Table 1 CSF autoimmune and paraneoplastic laboratory results

\begin{tabular}{|c|c|c|c|c|}
\hline Test & Interpretation & Technical result & Reference range & Methodology \\
\hline Anti-NR1 & Negative & Negative & Not applicable & IIFT \\
\hline Anti-alpha $3 \mathrm{AChR}$ & Negative & Negative & Not applicable & RIA \\
\hline Anti-GAD65 antibody & Positive & $>1: 4800$ & $\begin{array}{l}\text { Negative }<1: 600 \\
\quad \text { Borderline 1:600-1:1200, } \\
\text { Positive }>1: 1200\end{array}$ & ELISA \\
\hline Anti-LGI & Negative & Negative & Not applicable & IIFT \\
\hline Anti-VGCC & Negative & $<55$ & $\begin{array}{l}\text { Negative }<71, \text { Borderline } 71-140, \\
\text { Positive }>140(\mathrm{pmol} / \mathrm{L})\end{array}$ & RIA \\
\hline Anti-VGKC & Negative & $<100$ & $\begin{array}{l}\text { Negative }<112, \text { Borderline } 112-269, \\
\text { Positive }>269(\mathrm{pmol} / \mathrm{L})\end{array}$ & RIA \\
\hline Anti-CASPR2 & Negative & Negative & Not applicable & IIFT \\
\hline Anti-amphiphysin & Negative & $<1: 100$ & Serum $<1: 100$ & Nanoliter scale immunoassay \\
\hline Anti-CV2 & Negative & $<1: 100$ & Serum $<1: 100$ & Nanoliter scale immunoassay \\
\hline Anti-Hu & Negative & $<1: 100$ & Serum $<1: 100$ & Nanoliter scale immunoassay \\
\hline Anti-Ma and Anti-Ta & Negative & $<1: 100$ & Serum $<1: 100$ & Nanoliter scale immunoassay \\
\hline Anti-recoverin & Negative & $<1: 50$ & Serum $<1: 50$ & Nanoliter scale immunoassay \\
\hline Anti-RI & Negative & $<1: 50$ & Serum $<1: 50$ & Nanoliter scale immunoassay \\
\hline Anti-Yo & Negative & $<1: 200$ & Serum $<1: 200$ & Nanoliter scale immunoassay \\
\hline Anti-Zic4 & Negative & $<1: 50$ & Serum $<1: 50$ & Nanoliter scale immunoassay \\
\hline
\end{tabular}

found to be more effective. Similarly, in our case, clinical response was ultimately achieved with rituximab following ineffective treatment with steroids, IVIg, and PLEX, consistent with the aforementioned reports of autoimmune-mediated $\mathrm{SE}$ in the literature.

On initial presentation, it can be difficult to distinguish between NORSE of autoimmune etiology versus infectious etiology. Patient prognosis remains highly dependent on the underlying etiology, and treatment regimens differ significantly, making early suspicion in either direction of great importance. It has been suggested that autoimmune etiology is associated with certain clinical features such as younger age of onset $(<50)$, female sex, psychosis, and super-refractory SE, in addition to lymphocytic predominance in CSF. In contrast, other features such as high CSF portal protein, pleiocytosis and reduced glucose ratio are associated with infectious etiology [14]. Raising suspicion based on these factors early on in a hospital admission can help guide rapid initiation of appropriate therapy to achieve clinical response and neuroprotection. Notably, although CSF analysis can be helpful in this distinction, in the case of anti-GAD65 antibody-mediated autoimmune epilepsy, lack of response to IVIg and PLEX has been shown to be to typical $[16,17]$ and possibly diagnostic. Given that the prevalence of autoimmune and infectious status epilepticus are similar, factors that facilitate early clinical suspicion for either process are valuable for ICU physicians in the rapid initiation of appropriate empiric therapy.

Autoimmune etiology has recently been recognized as an important cause in epilepsy and in NORSE. In recent years, increasing numbers of auto-antibodies, which can be either autoimmune or paraneoplastic in nature, have been identified in patients with new-onset seizures. These auto-antibodies are involved in maintaining the balance between excitatory and inhibitory neurotransmission, underlying their role in seizure activity [14]. Antibodies associated with autoimmune epilepsy can be categorized into two groups: those against neuronal cell surface and synaptic proteins, and those against intracellular antigens.

Intracellular antigen-mediated autoimmune epilepsy (e.g., mediated by anti-Hu/ANNA-1, Ma2/Ta, CV2/CRMP5, and GAD65) is frequently paraneoplastic in nature with the exception of purely GAD65-related epilepsy [15, 20]. GAD65mediated epilepsy is also typically more refractory to therapy, as demonstrated in our case, and carries a poorer prognosis when compared with neuronal cell surface/synaptic autoimmune epilepsy (e.g., mediated by anti-NMDAR, GABAR, LGI1 [15]). These elements of GAD65-mediated autoimmune epilepsy are consistent with our clinical scenario in addition to the fact that our workup was negative for paraneoplastic causes. Furthermore, it has been suggested in the literature that in contrast to other autoimmune-mediated epilepsies, GAD65 antibody-mediated epilepsy is less responsive to standard AEDs and to immune therapy with steroids, IVIg, and PLEX, and that treatment with aggressive immunosuppressants such as rituximab is necessary to achieve clinical response $[16,17]$ as is demonstrated in our patient.

The pathogenic role of GAD65 is debated in the literature. Although the precise mechanism of pathogenicity remains unclear, it is suggested that intrathecal synthesis of GAD65 antibody leads to the degeneration of GABAergic neurons that 
consequently release cytoplasmic proteins into the CSF eliciting an immune response [18]. As GAD is expressed by GABAergic neurons and is an enzyme involved in the conversion of glutamate to GABA, the inhibition of GAD via an autoimmune process could result in excessive excitatory neurotransmission, ultimately resulting in lowering of the seizure threshold $[18,19]$.

The management of autoimmune SE requires a balance of AEDs and IV anesthetics for burst suppression while the immunosuppressant therapy treats the underlying cause of NORSE. AED selection in GAD65 epilepsy is similar to generalized clinically acceptable principles, that is, to achieve seizure control and tolerability. The challenges of therapy for burst suppression require frequent patient monitoring in a critical care setting, continuous subjective interpretation of EEG, and the careful titration of IV anesthetics. Further monitoring must be done to avoid AEDs and IV anesthetics at dangerous levels or the development of adverse side effects. Furthermore, there are consequences to prolonged high-dose infusion for each of these IV anesthetic agents. For example, due to high-dose propofol infusions for multiple days, the patient was screened for propofol infusion syndrome by following creatinine protein kinase (CPK) levels and lactate levels. In addition, treatment with phenobarbital requires the monitoring of a basic metabolic panel (BMP) for hypokalemia and liver function tests (LFTs) for hepatic dysfunction. Of note, phenobarbital is a CYP P450 inducer and affects the serum levels of other AEDs, in this case phenytoin. It is important to monitor serum drug levels to ensure therapeutic levels of phenytoin are maintained while phenobarbital is being administered.

In conclusion, this challenging case of NORSE in a previously healthy young adult emphasizes the importance of rapid recognition of autoimmune etiology as well the achievement of burst suppression in SE refractory to multiple therapeutic approaches. Despite the high-mortality prognosis, the prolonged duration of the epileptic state, and the difficulty in maintaining burst suppression, the clinical evolution resulted in complete neurological recovery.

Authors' contributions LB: This author contributed to original writing, revisions, editing, and conceptual design.

PH: This author contributed to original writing, revisions, editing, and conceptual design.

JS: This author contributed to original writing, revisions, editing, and conceptual design.

\section{Compliance with Ethical Standards}

Conflict of Interest The authors declare that they have no conflicts of interest.

Ethical Approval A written HIPAA authorization to use/disclose existing protected health information was obtained.

Informed Consent Informed consent was obtained from all individual participants included in the study.

\section{References}

1. Mayer SA, Claassen J, Lokin J, Mendelsohn F, Dennis LJ, Fitzsimmons BF. Refractory status epilepticus: frequency, risk factors, and impact on outcome. Arch Neurol. 2002;59:205.

2. Hocker SE. Status Epilepticus. Neurocrit Care. 2015;21:1362-83.

3. Vasile B, Rasulo F, Candiani A, Latronico N. The pathophysiology of propofol infusion syndrome: a simple name for a complex syndrome. Intensive Care Med. 2003;29:1417-25.

4. Pugin D, Foreman B, De Marchis GM, Fernandez A, Schmidt JM, Czeisler BM, et al. Is pentobarbital safe and efficacious in the treatment of super-refractory status epilepticus: a cohort study. Crit Care. 2014;18(3):R103.

5. Zeiler FA, Teitelbaum J, Gillman LM, West M. NMDA antagonists for refractory seizures. Neurocrit Care. 2014;20(3):502-13.

6. Avidan MS, Maybrier HR, Abdallah AB, Jacobsohn E, Vlisides $\mathrm{PE}$, Pryor KO, et al. Intraoperative ketamine for prevention of postoperative delirium or pain after major surgery in older adults: an international, multicentre, double-blind, randomised clinical trial. Lancet. 2017;390(10091):267-75.

7. Chen JW, Wasterlain CG. Status epilepticus: pathophysiology and management in adults. Lancet Neurol. 2006;5(3):246-56.

8. Cervenka MC, Hocker S, Koenig M, et al. Phase I/II multicenter ketogenic diet study for adult superrefractory status epilepticus. Neurology. 2017;88(10):938-43.

9. Schmitt FC, Buchheim K, Meierkord H, Holtkamp M. Anticonvulsant properties of hypothermia in experimental status epilepticus. Neurobiol Dis. 2006;23:689-96.

10. Legriel S, Lemiale V, Schenck M, et al. Hypothermia for neuroprotection in convulsive status epilepticus. N Engl J Med. 2016;375: 2457-67.

11. Lowenstein DH, Alldredge BK. Status Epilepticus. N Engl J Med. 1998;338:970-6.

12. Cabezudeo-Garcia P, Mena-Vazquez N, Villagran-Garcia M, Serrano-Castro PJ. Efficacy of antiepileptic drugs in autoimmune epilepsy: a systematic review. Seizure. 2018;59:72-6.

13. Makela KM, Aki H, Antti B, Jukka P. Clinical management of epilepsy with glutamic acid decarboxylase antibody positivity: the interplay between immunotherapy and anti-epileptic drugs. Front Neurol. 2018;9:579.

14. Lin CH, Lu YT, Ho CJ, Shih FY, Tsai MH. The different clinical features between autoimmune and infectious status epilepticus. Front Neurol. 2019;10:25.

15. Bien CG, Vincent A, Barnett MH, Becker AJ, Blümcke I, Graus F, et al. Immunopathology of autoantibody-associated encephalitides: clues for pathogenesis. Brain. 2012;135:1622-38.

16. Baroncini D, Spagnolo F, Sarro L, Comi G, Volonte MA. A complex case of anti-GAD antibody-related syndrome treated with Rituximab. Neurol Sci. 2013;34:1847-9.

17. Planche V, Marques A, Ulla M, Ruivard M, Durif F. Intravenous immunoglobulin and rituximab for cerebellar ataxia with glutamic acid decarboxylase autoantibodies. Cerebellum. 2014;13:318-22.

18. Solimena M, Folli F, Denis-Donini S, Comi GC, Pozza G, De Camilli $\mathrm{P}$, et al. Autoantibodies to glutamic acid decarboxylase in a patient with stiff-man syndrome, epilepsy, and type I diabetes mellitus. N Engl J Med. 1988;318:1012-20.

19. Kanter IC, Huttner HB, Staykov D, Biermann T, Struffert T, Kerling F, et al. Cyclophosphamide for anti-GAD antibody-positive refractory status epilepticus. Epilepsia. 2008;49:914-20.

20. Dalmau J. Status epilepticus due to paraneoplastic and nonparaneoplastic encephalitides. Epilepsia. 2009;50:58-60.

Publisher's Note Springer Nature remains neutral with regard to jurisdictional claims in published maps and institutional affiliations. 